\title{
Apoio social online entre cuidadores de crianças e jovens: revisão integrativa em rede da literatura
}

\section{Apoyo social en línea entre cuidadores de niños y jóvenes: revisión integrativa de la literatura en red}

\section{Online social support among caregivers of children and youth: networked integrative literature review}

\author{
Thiago da Silva Dias ${ }^{1}$, ORCID 0000-0002-8514-9124 \\ Fernando Augusto Ramos Pontes ${ }^{2}$, ORCID 0000-0001-9569-943X \\ Hugo Cardoso de Almeida ${ }^{3}$, ORCID 0000-0002-2697-7085 \\ Talita dos Santos Rosa ${ }^{4}$, ORCID 0000-0001-8997-6584 \\ Maély Ferreira Holanda Ramos ${ }^{5}$, ORCID 0000-0001-6150-6345 \\ Simone Souza da Costa Silva ${ }^{6}$, ORCID 0000-0003-0795-2998 \\ ${ }^{1,2,3,6}$ Núcleo de Teoria e Pesquisa do Comportamento, Universidade Federal do Pará. Brasil \\ ${ }^{4}$ Escola de Enfermagem de Ribeirão Preto, Universidade de São Paulo. Brasil \\ ${ }^{5}$ Instituto de Ciências da Educação, Universidade Federal do Pará. Brasil
}

Resumo: A Internet pode ser uma fonte de apoio social que auxilia no aprimoramento de competências parentais. Objetivou-se analisar o panorama internacional das pesquisas que investigaram apoio social online entre cuidadores de crianças e jovens. $\mathrm{O}$ estudo consiste em uma Revisão Integrativa em Rede da Literatura (RIRL) que aborda a literatura como uma rede de associação de variáveis e a analisa com base na Teoria dos Grafos. A busca ocorreu em duas bases de dados e os estudos foram filtrados por meio de dois testes de relevância. Foram resgatados 36 artigos, cujos resultados foram convertidos em 53 variáveis, que foram categorizadas conforme suas relações. Considerando-se a recorrência de relações entre as variáveis, conclui-se que "Participação em grupos online" favorece o "Apoio social", a "Conexão com outras pessoas na mesma situação" e o surgimento do "Senso de comunidade". Outros achados são discutidos de acordo com as medidas de redes.

Palavras-chave: análise de redes, apoio social online, cuidadores, grupos de apoio, revisão integrativa

\begin{abstract}
The Internet may be a source of social support that promotes the improvement of parental competency. The objective of this study was to analyze the international panorama regarding research on online social support among caregivers of children and youth. This study comprises a Networked Integrative Review of Literature (NIRL) that approaches the literature as an associative network of variables and analyzes it based on the Theory of Graphs. Data search was conducted in two databases and the studies were filtered based on two relevance tests. This NIRL comprises 36 articles, whose results were converted into 53 variables that were categorized according to their relationship. Considering the recurrence of relationships among the variables, it is concluded that "Participation in online groups" promotes "Social support", "Connection to other people in the same situation" and the emergence of a "Sense of Community". Other findings are discussed based on the network measures.
\end{abstract}

Keywords: caregivers, network analysis, integrative review, online social support, self-help groups 
Resumen: La internet puede ser una fuente de apoyo social que ayuda a mejorar las competencias parentales. Se objetivó analizar el panorama internacional de los estudios acerca de apoyo social en línea entre cuidadores de niños y jóvenes. El estudio consiste en una Revisión Integrativa de la Literatura en Red (RILR), que aborda a la literatura como una red asociativa de variables y la analiza basada en la Teoría de los Grafos. La búsqueda se realizó en dos bases de datos y los estudios fueron filtrados a partir de dos pruebas de relevancia. Se recuperaron 36 estudios, cuyos resultados fueron convertidos en 53 variables que fueron categorizadas según sus relaciones. Considerando la recurrencia de relaciones entre las variables, se concluye que "Participación en grupos en línea" favorece "Apoyo social", "Conexión con otras personas en la misma situación" y lo surgimiento de un "Sentido de comunidad". Otros hallazgos son discutidos basados en las medidas de red.

Palabras clave: análisis de redes, apoyo social en línea, cuidadores, grupos de autoayuda, revisión integrativa

Recebido: 27/09/2018

Aceito: $12 / 03 / 2020$

Como citar este artigo:

Dias, T.S., Pontes, F.A.R., Almeida, H.C., Rosa, T.S., Ramos, M.F.H., \& Silva, S.S.C. (2020). Apoio social online entre cuidadores de crianças e jovens: revisão integrativa em rede da literatura. Ciencias Psicológicas, 14(1), e-2060. doi: https://doi.org/10.22235/cp.v14i1.2160

Correspondência: Thiago da Silva Dias, Fernando Augusto Ramos Pontes. Universidade Federal do Pará, Núcleo de Teoria e Pesquisa do Comportamento, Programa de Pós-Graduação em Teoria e Pesquisa do Comportamento, Rua Augusto Corrêa, 01, CEP 66075-110, Belém, Pará, Brasil. E-mails: thiagodias.to@gmail.com; farp1304@gmail.com

\section{Introdução}

Apoio social (AS) é definido como um processo que envolve a troca de recursos interpessoais entre, pelo menos, dois indivíduos, com o objetivo de suprir diferentes tipos de necessidade (Lin, Hsu, Cheng, \& Chiu, 2015). Estes recursos são necessários, principalmente, em momentos de transições, tais como as ocorridas na adaptação ao papel de cuidador, incluindo mudanças e ajustes em aspectos físicos, psicossociais e materiais/financeiros (Epifanio, Genna, De Luca, Roccella, \& La Grutta, 2015). Dessa forma, compreende-se que cuidadores necessitam de AS para lidar com as transições inerentes ao seu papel.

Neste contexto, Wei et al. (2012) indicam que os cuidadores têm, principalmente, a necessidade de apoio emocional e informacional, ou seja, obtenção de conhecimento, sugestões, orientações, empatia e encorajamento para se sentirem mais confiantes nas tarefas de cuidado. O acesso a estes tipos de AS pode minimizar os efeitos do estresse advindo das tarefas de cuidado sobre a saúde dos cuidadores, visto que está negativamente relacionado com os níveis de estresse percebido e fisiológico, com o aumento patológico da pressão arterial e com sintomas de depressão (Cantwell, Muldoon, \& Gallagher, 2014; Gallagher \& Whiteley, 2012; Lovell, Moss, \& Wetherell, 2012).

Porém, o acesso ao AS pode ser influenciado pelo baixo nível de participação social presencial destes sujeitos, visto que as interações e, consequentemente, as suas fontes de AS comumente, são restritas ao círculo mais íntimo de contatos sociais, incluindo familiares, amigos e colegas de trabalho (Thuy \& Berry, 2013). Esta diminuição na participação social pode ser 
minimizada por meio da Internet, que pode viabilizar AS, em detrimento das limitações de tempo dos cuidadores. Destaca-se que plataformas online têm uma velocidade de informação muito maior do que grupos presenciais, favorecendo a viabilização de informações por meio de interações multimodais, incluindo fotos, vídeos, documentos compartilhados, entre outros (Lee \& Kvasny, 2014).

Apesar do crescimento no número de estudos que investigam a viabilidade de apoio social online (ASO), não foram identificadas revisões de literatura (RL) com enfoque concomitante na variável e no público deste estudo, embora tenham sido recuperadas RL acerca do uso de relações na internet para obter informações relacionadas à saúde e ao cuidado parental (Niela-Vilén, Axelin, Salanterä \& Melender, 2014; Park, Kim, \& Steinhoff, 2016).

Neste contexto, destaca-se que as revisões integrativas da literatura (RIL) são caracterizadas por uma abordagem ampla que objetiva sintetizar resultados obtidos em pesquisas sobre um tema (Mendes, Silveira, \& Galvão, 2008). Este método permite a inclusão simultânea de pesquisas quase-experimentais e experimentais, teóricas, empíricas, quantitativas e qualitativas (Whittemore \& Knafl, 2005), proporcionando uma compreensão mais ampla do tema.

A apresentação dos resultados da revisão, conforme Whittemore e Knafl (2005), deve ser efetuada por meio de um modelo abrangente, que demonstre o processo de integração. Dessa forma, propõe-se a utilização da lógica da análise de redes (AR), instrumentada pela teoria dos grafos (TG), cujo potencial para fins de revisão da literatura foi demonstrado em estudos precursores (Ramos, Fernandez, Pontes, \& Silva, 2016). Neste estudo, este modelo foi denominado Revisão Integrativa em Rede da Literatura (RIRL) e está baseado na perspectiva de que o conjunto da literatura produz uma "rede de conhecimento" (Diallo, Lynch, Gore, \& Padilla, 2016) resultante das relações entre seus produtos de conhecimento.

Neste estudo, a rede de conhecimento é baseada nas relações entre as variáveis investigadas nos artigos, consideradas produtos de conhecimento. Deste modo, os artigos recuperados podem ser organizados por meio de um determinado sistema replicável de busca, a fim de relacionar as variáveis estudadas e, com base em uma tabela de contingência, construir uma narrativa integrada em rede dos resultados dos artigos, desenvolvida com base na TG. Dessa forma, este estudo objetivou analisar o panorama internacional das pesquisas que investigaram ASO entre cuidadores de crianças e jovens (CCJ) com base em um modelo de RIRL, utilizando a TG como instrumentação para a integração dos achados dos estudos. Especificamente, objetiva-se descrever as características dos estudos nesta área, identificar e descrever as principais variáveis abordadas pelos estudos e as suas relações com a obtenção de ASO por CCJ, verificar a aplicabilidade do uso da TG como instrumento de organização da apresentação dos resultados de uma revisão integrativa e caracterizar a rede de conhecimento composta pelas relações entre as variáveis dos estudos.

\section{Materiais e Método}

A RIRL apresenta elementos de Revisão Sistemática da Literatura (RSL), no sentido que possui um sistema de coleta de dados com métodos de seleção dos artigos e tratamento de dados que possibilita a replicação. Adicionalmente, a sua análise é baseada na nova ciência das redes (Lewis, 2009), instrumentada pelos métodos de análise da TG. Em síntese, esta RIRL foi estruturada em cinco etapas, descritas a seguir.

Na primeira etapa, a questão de pesquisa foi estruturada com base na estratégia PVO População, Variáveis e Outcomes/Desfechos (Ramos et al., 2016). Dessa forma, a questão de pesquisa inclui os seguintes elementos: $\mathrm{P}$ - cuidadores de crianças e jovens; $\mathrm{V}$ - variáveis usadas para medir o "apoio social online" ou como decorrência dele; $\mathrm{O}$ - resultado verificado nos participantes, indicador da modificação ou relação estabelecida entre as variáveis.

Visando à sistematização da coleta e refinamento do conteúdo, foram pré-determinados os seguintes critérios de elegibilidade: termos de busca no assunto, resumo, palavras-chave e/ou 
título; publicação entre 2007 e 2016; artigos completos e disponíveis; idioma inglês/português/espanhol; publicação em periódicos avaliados por pares; pesquisa empírica; foco em CCJ; e foco em ASO.

$\mathrm{Na}$ segunda etapa, os portais dos Descritores em Ciências da Saúde (DeCs) e das Terminologias em Psicologia foram utilizados para a identificação de descritores condizentes com os elementos "P (população)" e "V (variáveis)". Ressalta-se que termos qualificadores "online", "virtual" e "Internet" foram adicionados aos descritores "V" (tipo de AS). Além disso, a utilização do símbolo (*) visa obter resultados para os descritores no singular e no plural.

Posteriormente, a plataforma "ScienceDirect" e o "Portal de Periódicos da Capes", ambos líderes na indexação de literatura científica internacional, foram selecionados para busca de artigos. No âmbito das duas plataformas, operadores booleanos foram aplicados aos componentes P e V da estratégia PVO: (P) AND (V). Desse modo, conforme exemplificado a seguir, foram geradas três estratégias de busca, de acordo com idiomas selecionados:

- (caregiver* OR parent* OR mother* OR father* OR famil*) AND (internet support group* OR online support group* OR virtual support group* OR internet self-help group* OR online self-help group* OR virtual self-help group* OR internet social support OR online social support OR virtual social support OR internet support communit* OR online support communit* OR virtual support communit* OR internet self-help communit* OR online selfhelp communit* OR virtual self-help communit*)

As mesmas estratégias foram aplicadas à plataforma Cochrane Library, porém não foram identificadas revisões que contemplassem a variável ASO e, concomitantemente, CCJ.

$\mathrm{Na}$ terceira etapa, objetivando à seleção de estudos, foram elaborados dois testes de relevância. O Teste de Relevância 1 (TR1) foi executado pelo pesquisador principal com base na leitura das seções de métodos e resultados dos artigos elegíveis, visando verificar a adequação dos artigos à questão de pesquisa. Posteriormente, os artigos considerados elegíveis foram submetidos ao Teste de Relevância 2 (TR2), que incluiu a leitura dos artigos na íntegra por dois avaliadores independentes. Um cálculo de concordância quanto ao parecer dos avaliadores foi efetuado, de modo que foram considerados somente os aprovados de forma unânime.

$\mathrm{Na}$ quarta etapa, com base em uma leitura exploratória, foi efetuada a caracterização dos artigos quanto idioma, local, ano de publicação, abordagem, métodos de coleta e análise de dados, variáveis estudadas entre outros aspectos relevantes.

Finalmente, na quinta etapa, com base no elemento "O (outcomes/resultados)", a AR e a TG foram utilizadas para integrar os dados da literatura recolhida. Assim, foi realizada uma leitura detalhada dos resultados dos artigos selecionados, analisando as variáveis apresentadas e as suas respectivas relações, que foram dispostas em uma tabela de contingência, respectivamente, como vértices pareados e as arestas correspondentes. Estes dados foram tratados pelo programa NodeXL (Yep \& Shulman, 2014), onde foi construída uma rede de relações entre as variáveis identificadas, bem como as mesmas foram organizadas em conglomerados com base no Algoritmo CNM (Clauset, Newman e Moore, 2004), visando à caracterização dos campos de relações na rede. De acordo com Clauset et al. (2004) o algoritmo CNM procura um particionamento do grafo em comunidades ou conglomerados de maneira que seja maximizada a modularidade, que se refere à uma estrutura específica de rede que otimiza a divisão na medida em que há muitas arestas dentro das comunidades e apenas algumas entre elas. 


\section{Resultados e Discussão}

Os procedimentos de busca resultaram no resgate de 1471 artigos, dos quais, após a remoção de duplicatas, restaram 571 artigos, que, após submetidos ao TR1 e TR2, foram reduzidos a 36 artigos elegíveis para a RIRL.

\section{Caracterização Geral}

Todos os artigos foram publicados em língua inglesa. Quanto às demais características dos estudos, estas são apresentadas na Tabela 1.

Tabela 1

Características dos estudos recuperados

\begin{tabular}{|c|c|c|c|}
\hline Característica & Categorias & Total & Percentual (\%) \\
\hline \multirow{4}{*}{ Local $^{1}$} & América do Norte & 21 & 58.3 \\
\hline & Europa & 10 & 27.8 \\
\hline & Oceania & 7 & 19.4 \\
\hline & Ásia & 1 & 2.8 \\
\hline \multirow{3}{*}{ Ano de publicação } & $2007-2010$ & 4 & 11.1 \\
\hline & 2011-2013 & 15 & 41.7 \\
\hline & 2014-2016 & 17 & 47.2 \\
\hline \multirow{3}{*}{ Abordagem } & Qualitativa & 27 & 75.0 \\
\hline & Quantitativa & 5 & 13.9 \\
\hline & Quanti-qualitativa & 4 & 11.1 \\
\hline \multirow{2}{*}{ Coleta de dados ${ }^{2}$} & Exclusivos & 26 & 72.2 \\
\hline & Mistos & 10 & 27.8 \\
\hline \multirow{2}{*}{ Análise de dados ${ }^{3}$} & Exclusivos & 30 & 83.3 \\
\hline & Mistos & 6 & 16.7 \\
\hline \multirow{3}{*}{ Gênero dos participantes } & Feminino & 16 & 44.4 \\
\hline & Masculino & 3 & 8.3 \\
\hline & Inespecífico $^{4}$ & 17 & 47.2 \\
\hline \multirow{2}{*}{ Ambiente online ${ }^{5}$} & Específicos & 28 & 77.8 \\
\hline & Genéricos & 8 & 22.2 \\
\hline \multirow{2}{*}{ Enfoque do estudo } & Parentalidade típica & 15 & 41.7 \\
\hline & Parentalidade e deficiência & 21 & 58.3 \\
\hline \multirow{3}{*}{ Ênfase do estudo } & Descrição de experiências & 30 & 83.3 \\
\hline & Intervenção & 5 & 13.9 \\
\hline & Mistos & 1 & 2.8 \\
\hline
\end{tabular}

Legenda: ${ }^{1}$ há sobreposição na porcentagem decorrente de estudos multinacionais; ${ }^{2}$ uso exclusivo ou combinado de extrações de dados e questionários presenciais ou online; ${ }^{3}$ uso exclusivo ou combinado de estatística descritiva e análise de conteúdo ou de discurso; ${ }^{4}$ genitores ou familiares de modo geral (majoritariamente mulheres); ${ }^{5}$ apresentação de fóruns, grupos, chats, listas de e-mail e blogs específicos ou descrição genérica da experiência de apoio social online.

\section{Integração dos Resultados}

Nesta RIRL, foram identificadas 53 variáveis diferentes (vértices), que estabeleciam oito tipos de relação (arestas): "correlações positivas e negativas"; "escores maiores $(>)$ e menores $(<)$ "; "relações diretas e inversas"; "favorece"; e "reduz". Nesta rede de variáveis, foram contabilizadas 269 arestas entre os vértices, das quais 199 foram identificadas em mais de um artigo. 
Com base no algoritmo CNM, as variáveis foram organizadas em conglomerados (Figuras 1-5). Este algoritmo particiona o grafo em conglomerados para maximizar a modularidade, ou seja, otimiza a divisão na medida em que há muitas arestas dentro dos grupos e apenas algumas entre eles (Clauset et al, 2004).

Há cinco grupos principais (G1 a G5), que integram uma rede composta de apenas um componente, de modo que todas as variáveis estão de alguma forma conectadas, ou seja, não há subgrupos desconectados. Dentre os grupos, o G1 (Figura 1) apresenta o maior número de variáveis envolvidas (16), contudo apresenta a menor densidade (0.075), enquanto o G5 (Figura 5) apresenta menos variáveis (7) e a maior densidade (0.190). A densidade de um grafo, variando entre 0 e 1, está relacionada à quantidade de ligações realizadas, de forma que redes mais densas contêm mais ligações entre os vértices (Hanneman \& Riddle, 2005). Este fator tem uma implicação no funcionamento integrado da rede, de forma que a ampliação ou incremento em determinadas áreas tendem a atingir as outras. Assim, a despeito de ser uma rede de somente um componente, grupos com maior densidade, tais como o G5, apresentam maior intercâmbio de relacionamento de variáveis entre si.

As relações entre variáveis componentes de grupos distintos são mostradas nas Figuras 15, genericamente, em forma de linhas cinzas. A espessura das arestas reflete a frequência de ocorrência da relação e o tamanho dos vértices indica a respectiva centralidade de intermediação (CI), ou seja, o grau em que uma variável estabelece pontes entre outros subconjuntos de variáveis.

A maioria dos grupos mantêm intensa relações entre si, a exceção do G3 (nenhuma relação direta com G4, conforme Figura 3) e do G4 (baixa relação com G2 e com G5, conforme Figura 4). A integração da rede de variáveis em um componente implica, supostamente, na possibilidade de transições entre conceitos e variáveis, de modo que, não há barreiras teóricas evidentes ou que possam ser suplantadas. Esta suposição pode ser verificada na ausência de incongruência dos achados nos artigos recuperados quanto a associações entre variáveis, de modo que na maioria das vezes a relação entre elas tendem a ser semelhantes, se complementam e, quando não, são na mesma direção. 


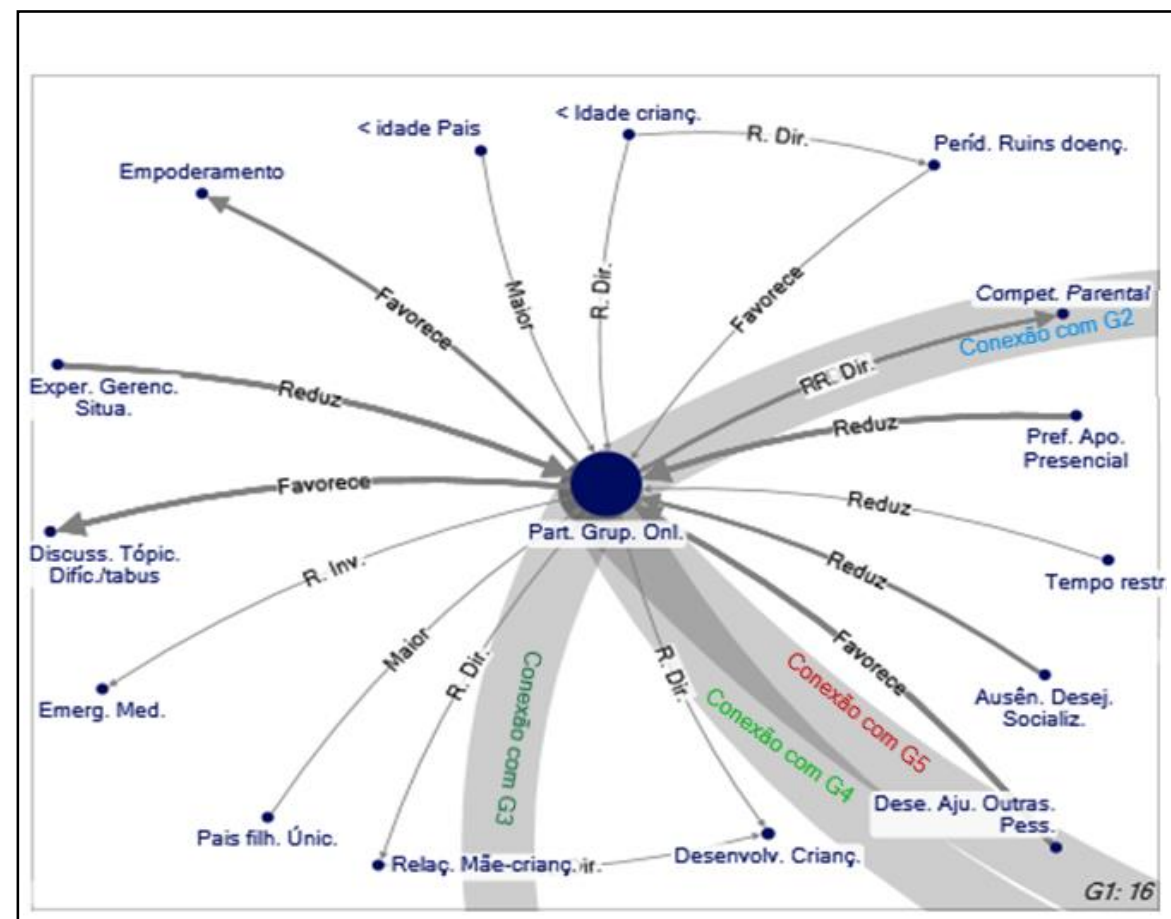

Figura 1 .

Subgrupo 1 da rede de relações de variáveis

Legenda: Espessura das linhas = quantidade de ligações entre variáveis/grupos. Direção das setas = direção da relação. Relações entre variáveis $=$ favorece, reduz, maior, R. Dir. (relação direta), R. Inv. (relação inversa). Lista de variáveis (abreviadas para otimizar o espaço) $=1$-pais de filho único, 2-relação mãecriança, 3-desenvolvimento da criança, 4-desejo de ajudar outras pessoas, 5ausência do desejo de socialização, 6-tempo restrito, 7-preferência por apoio presencial, 8-competências parentais, 9-períodos ruins da doença, 10-maior idade da criança, 11-maior idade dos pais, 12-empoderamento, 13-experiência no gerenciamento da situação, 14-discussão de tópicos difíceis/tabus, 15emergências médicas, 16-participação em grupos online. 


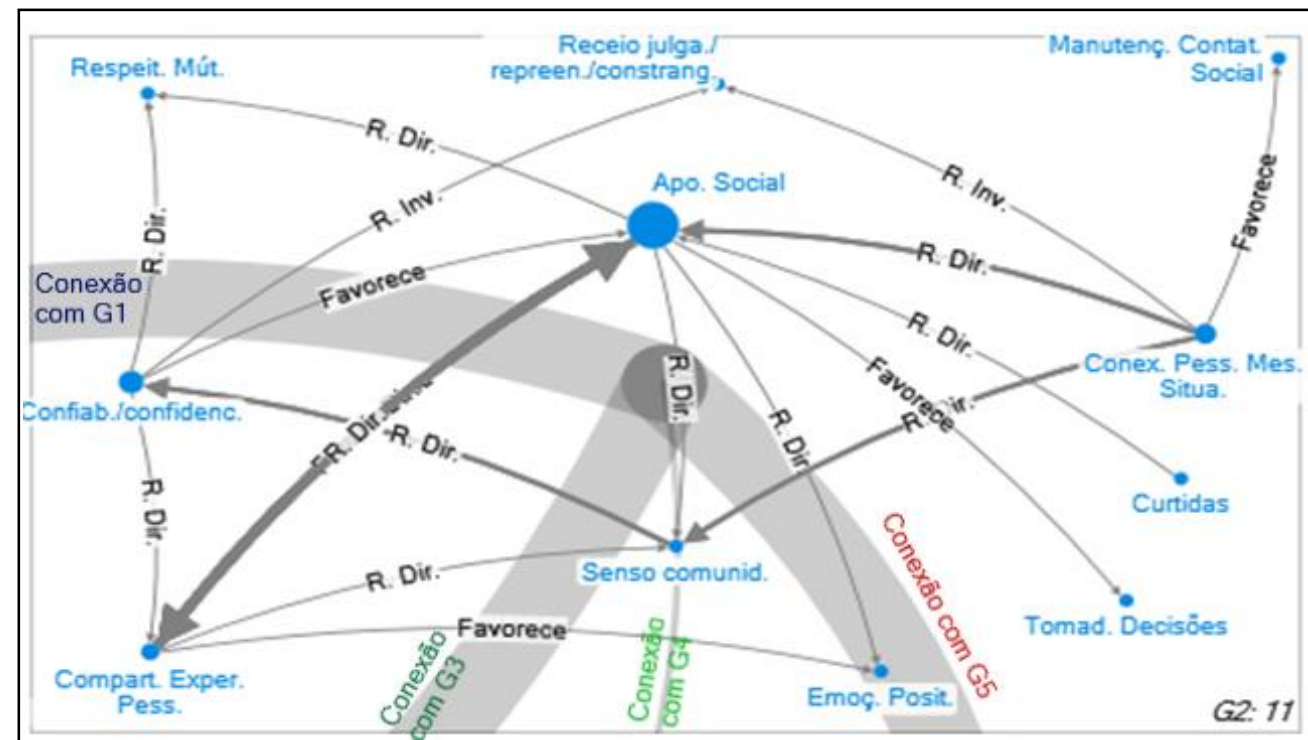

Figura 2.

Subgrupo 2 da rede de relações de variáveis

Legenda: Espessura das linhas = quantidade de ligações entre variáveis/grupos. Direção das setas $=$ direção da relação. Relações entre variáveis $=$ favorece, $R$. Dir. (relação direta), R. Inv. (relação inversa). Lista de variáveis (abreviadas para otimizar o espaço) $=1$ compartilhamento de experiências pessoais, 2-emoções positivas, 3-tomada de decisões, 4-senso de comunidade, 5-curtidas, 6-confidencialidade/confiabilidade, 7-conexão com outras pessoas na mesma situação, 8-apoio social, 9-respeito mútuo, 10-receio de julgamento/repreensão/constrangimento, 11-manutenção de contato social. 


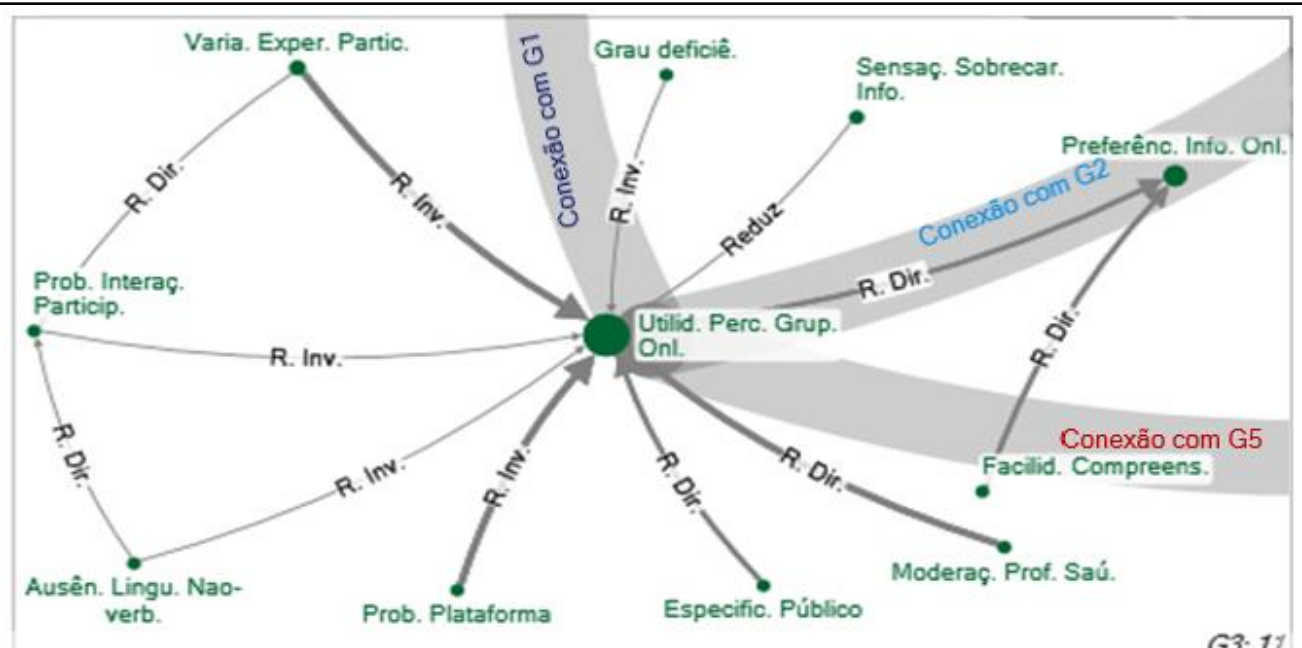

Figura 3.

Subgrupo 3 da rede de relações de variáveis

Legenda: Espessura das linhas = quantidade de ligações entre variáveis/grupos. Direção das setas $=$ direção da relação. Relações entre variáveis $=$ reduz, R. Dir. (relação direta), R. Inv. (relação inversa). Lista de variáveis (abreviadas para otimizar o espaço) $=1$-ausência de linguagem não-verbal, 2-problemas na plataforma, 3-especificidade do público, 4moderação por profissionais de saúde, 5-facilidade de compreensão, 6-utilidade percebida do grupo online, 7-problemas na interação entre os participantes, 8-variação na experiência dos participantes, 9-grau de deficiência da criança, 10-sensação de sobrecarga de informações, 11-preferência por informações online. 


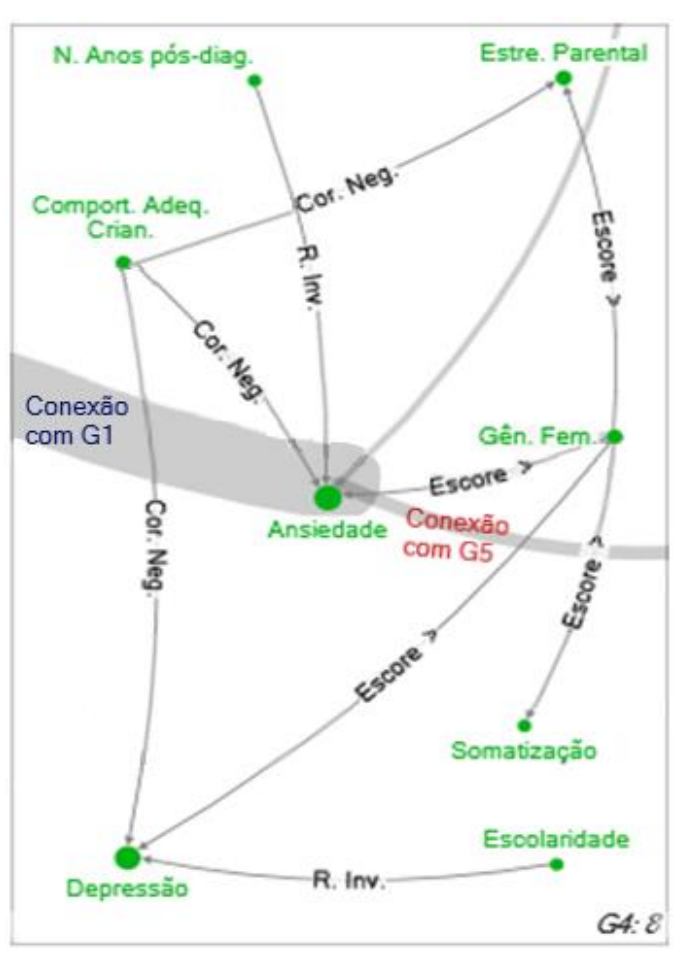

Figura 4.

Subgrupo 4 da rede de relações de variáveis

Legenda: Espessura das linhas = quantidade de ligações entre variáveis/grupos. Direção das setas = direção da relação. Relações entre variáveis $=$ Cor. Neg. (correlação negativa), escore > (escore maior), R. Inv. (relação inversa). Lista de variáveis (abreviadas para otimizar o espaço) $=1$-depressão, 2-escolaridade, 3-somatização, 4ansiedade, 5-gênero feminino, 6-comportamentos adequados da criança, 7-número de anos pós-diagnóstico, 8 -estresse parental. 


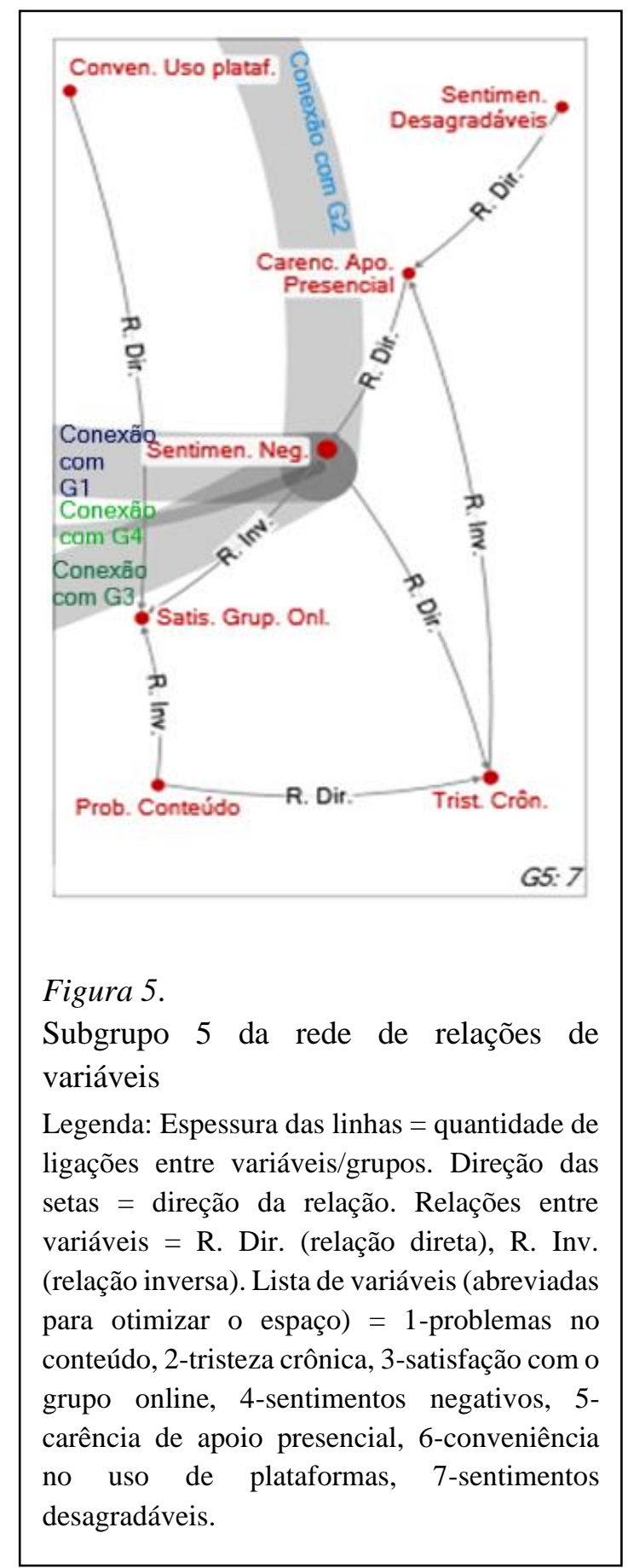

\section{Relações entre as Variáveis}

Com base nas relações verificadas nas Figuras 1-5, foi elaborada uma apresentação narrativa integrada dos resultados, a fim de demonstrar o funcionamento em rede das variáveis. Para fins didáticos, serão mostradas, prioritariamente, as relações com maior destaque em termos de intermediação e recorrência de associação

De uma perspectiva geral, os pais de crianças e jovens relatam o recebimento e a viabilização de "Apoio social" por meio da "Participação em grupos online" (Asiodu, Waters, Dailey, Lee, \& Lyndon, 2015; Binford Hopf, Le Grange, Moessner, \& Bauer, 2013; Bragadóttir, 
2008; Cacioppo, Conway, Mehta, Krantz, \& Noon, 2016; Clifford \& Minnes, 2013; Coulson \& Greenwood, 2012; Craig \& Johnson, 2011; Evans, Donelle, \& Hume-Loveland, 2012; Gabbert, Metze, Bührer, \& Garten, 2013; Glenn, 2015; Hudson, Campbell-Grossman, \& Hertzog, 2012; Johnson, 2015; Martin et al., 2014; Martin et al., 2016; Mustafa, Short, \& Fan, 2015; Nolan, Hendricks, \& Towell, 2015; Oprescu, Campo, Lowe, Andsager, \& Morcuende, 2013; Perrone, Carmody, Philipson, \& Greeley, 2015; Reinke \& Solheim, 2015; Roffeei, Abdullah, \& Basar, 2015).

Neste contexto, a participação online viabiliza, principalmente, a obtenção/promoção de informações e esclarecimento de dúvidas sobre aspectos relacionados à parentalidade, tanto em casos de crianças típicas como em situações nas quais a criança tem algum tipo de condição de saúde. Estas informações auxiliam no cuidado com a criança/jovem e no "Gerenciamento da situação" (Brady \& Guerin, 2010; Glenn, 2015; Hall \& Irvine, 2009; Johnson, 2015; Kirk \& Milnes, 2016; Oprescu et al., 2013; Parry, Glover, \& Mulcahy, 2013; Reinke \& Solheim, 2015; Salzmann-Erikson \& Eriksson, 2013; Sullivan, 2008; Valtchanov, Parry, Glover, \& Mulcahy, 2014). Além disso, estes sujeitos reportam a existência de relações de apoio emocional e à autoestima, acarretando em diminuição de "Sentimentos negativos" por meio da "Conexão com pessoas na mesma situação" (Appleton, Fowler, \& Brown, 2014; Brady \& Guerin, 2010; Gabbert et al., 2013; Morris \& Bertram, 2013; Oprescu et al., 2013; Pedersen \& Lupton, in press; Valtchanov et al., 2014).

Consonantemente, a análise de experiências de "Participação em grupos online" em plataformas com foco em temáticas específicas (maternidade, depressão pós-parto, autismo, asma, etc.) indicou que a "Conexão com pessoas na mesma situação" no ambiente online pode promover a troca de informações úteis sobre aspectos relacionados ao papel parental entre outros tipos de "Apoio social" online entre pais de crianças típicas e/ou de crianças com diferentes condições de saúde (Brady \& Guerin, 2010; Clarke \& Van Ameron, 2015; Eriksson \& Salzmann-Erikson, 2013; Hall \& Irvine, 2009; Kirk \& Milnes, 2016; Nolan et al., 2015; Parry et al., 2013; Reinke \& Solheim, 2015; Sullivan, 2008; Valtchanov et al., 2014), o que se torna essencial em situações nas quais há "Carência de apoio presencial" (Clarke \& Van Ameron, 2015; Kirk \& Milnes, 2016; Stewart, Letourneau, Masuda, Anderson, \& McGhan, 2011).

Destaca-se que os resultados integrados do estudo indicam que a "Participação em grupos online" e, consequentemente, a obtenção de "Apoio social" estabelecem um relação recíproca com sentimentos de "Empoderamento", "Competência parental", "Respeito mútuo", "Manutenção de contato social", "Conexão com pessoas na mesma situação", "Tomada de decisões", "Senso de comunidade", sentimentos de "Confiabilidade/ confidencialidade", "Compartilhamento de experiências pessoais", entre outras variáveis que podem ser obtidas por meio da "Participação em grupos online" e que, reciprocamente, motivam os pais a participar dos grupos online (Appleton et al., 2014; Brady \& Guerin, 2010; Clarke \& Van Ameron, 2015; Eriksson \& Salzmann-Erikson, 2013; Fletcher \& StGeorge, 2011; Gabbert et al., 2013; Glenn, 2015; Hall \& Irvine, 2009; Johnson, 2015; Kirk \& Milnes, 2016; Mustafa et al., 2015; Nolan et al., 2015; Oprescu et al., 2013; Parry et al., 2013; Pedersen \& Lupton, in press; Porter \& Ispa, 2013; Reinke \& Solheim, 2015; Roffeei et al., 2015; Salzmann-Erikson \& Eriksson, 2013; Sullivan, 2008; Valtchanov et al., 2014).

Da mesma forma, a análise integrada dos dados mostrou que a "Utilidade percebida do grupo online" pelos usuários é um fator-chave para a "Participação em grupos online" (Cacioppo et al., 2016; Clifford \& Minnes, 2013; Jacobs, Boyd, Brennan, Sinha, \& Giuliani, 2016; Martin et al., 2016; Morris \& Bertram, 2013; Stewart et al., 2011), que pode ser influenciada por aspectos relativos à experiência de interação em ambientes online, incluindo "Problemas de conteúdo", "Problemas de interação entre participantes", "Variabilidade nas experiências dos participantes", entre outras variáveis (Asiodu et al., 2015; Cacioppo et al., 2016; Clifford \& Minnes, 2013; Coulson \& Greenwood, 2012; Gabbert et al., 2013; Glenn, 2015; Reinke \& Solheim, 2015; Stewart et al., 2011). 
O conjunto de artigos também indica que "Sentimentos negativos" podem, alternadamente, motivar ou diminuir a "Participação em grupos online". Assim, os cuidadores podem buscar interações online para suprir a "Carência de apoio presencial", minimizar sentimentos de solidão, entre outros fatores (Clarke \& Van Ameron, 2015; Kirk \& Milnes, 2016; Stewart et al., 2011). Porém, em outra perspectiva, experiências na plataforma que acarretam "Sentimentos negativos" associadas a uma baixa "Utilidade percebida do grupo online" podem diminuir a "Participação em grupos online" (Cacioppo et al., 2016; Martin et al., 2014).

$\mathrm{Na}$ amostra desta RIRL, mais da metade (58.3\%) dos estudos abordavam a experiência de pais cujos filhos tinham algum tipo de deficiência ou condição de saúde, o que evidencia a viabilidade de relações de "Apoio social" online para este público. Além disso, a "Variabilidade nas experiências dos participantes" foi apontada como um aspecto negativo do uso de plataformas online, ou seja, os cuidadores preferiam estar em grupos com maior "Especificidade do públicoalvo", incluindo pais cujas experiências fossem as mais semelhantes possíveis às suas. Este aspecto pode ser entendido como uma das características da homofilia, ou seja, a tendência de indivíduos de interagirem preferencialmente com pessoas com perfil similar a eles (Laniado, Volkovich, Kappler, \& Kaltenbrunner, 2016).

Em outro sentido, o conjunto de artigos evidenciou aspectos negativos/barreiras advindos da "Participação em grupos online", tais como "Problemas na plataforma", "Problemas de interação entre os participantes" e "Problemas de conteúdo", incluindo a postagem de conteúdos disruptivos para o grupo (assuntos relacionados à morte, tristeza e sofrimento), atraso/falta de repostas aos questionamentos uns dos outros, dificuldades de se expressar por meio de aparelhos eletrônicos - considerados impessoais e ausentes de linguagem corporal - e compartilhamento de informações complexas e/ou incorretas. Por fim, destaca-se que muitos participantes demandavam a participação de profissionais nas plataformas, a fim de assegurar a confiabilidade do conteúdo relacionado à saúde.

Outro aspecto importante se refere à relação diferencial entre o tipo de apoio e o evento disparador a ele relacionado. De acordo com Perrone et al. (2015) o apoio à autoestima estava relacionado a eventos positivos, como o alcance de um marco do desenvolvimento, a superação de uma dificuldade, entre outros. Em contrapartida, o apoio emocional estava relacionado a eventos negativos, como a perda de um filho, pioras no quadro clínico, diagnóstico recémrecebido, entre outros.

Destaca-se que os resultados desta RIRL ratificam os dados do estudo de Niela-Vilén et al. (2014) quanto ao fato de que mães representam os principais participantes em plataformas de ASO. Além disso, os dados corroboram quanto à perspectiva de que o sentimento de fazer parte de um grupo formado por pessoas com experiências similares é um fator-chave na participação de cuidadores em plataformas online, o que ratifica a ocorrência de um fenômeno de homofilia.

\section{Conclusões}

Nesta RIRL, a literatura encontrada refere-se a um conjunto de dados essencialmente internacional. Por meio da análise de rede foi possível extrair o padrão da "rede de conhecimento" na área de ASO entre CCJ. Neste sentido, a literatura da área apresenta um perfil integrado, caracterizado pela ausência de barreiras teóricas que condicionem fronteiras conceituais, o que foi reforçado pela inexistência de dados incongruentes. Por outro lado, os subcampos de pesquisa apresentam topologias de rede de conhecimento diferenciados, por exemplo o grupo 1 apresenta um perfil de rede mais centralizado e com menor densidade, em relação aos demais grupos.

Nesta "rede de conhecimento", alguns vértices apresentaram um maior poder de centralização, tal como "Participação de grupos online", "Apoio social", "Utilidade percebida do grupo online", "Ansiedade", "Depressão" e "Sentimentos negativos", as quais são variáveis que intermedeiam relações entre outras variáveis. Considerando a recorrência de achados presentes 
acima de cinco artigos e o grau de centralidade, é possível sintetizar a seguinte integração presente na literatura: 1) "Participação em grupos online" favorece o "Apoio social', a "Conexão com pessoas na mesma situação" e o surgimento do "Senso de comunidade"; 2) a presença do "Apoio social" apresenta uma relação direta bidirecional com o "Compartilhamento de experiências pessoais", apresenta uma relação inversa com "Sentimentos negativos" e uma relação direta com a "Preferência por informações online" e com a "Utilidade percebida do grupo online"; 3) A "Carência de apoio presencial" é um dos fatores que favorece a "Participação em grupos online".

Ressalta-se que a utilização de uma metodologia baseada em redes para efetuar a integração dos dados é uma das principais contribuições científicas desta RIRL, visto que esta abordagem dos dados favorece a geração de um referencial teórico integrado ou sistemicamente relacionado. Neste sentido, entende-se que a RIRL é uma perspectiva inovadora e promissora para integrar a literatura dispersa em dados de natureza quantitativa e qualitativa. Por intermédio da TG e dos elementos de AR, é possível obter medidas estruturais da rede total recuperada, das relações entre variáveis e dos papéis de cada variável no conjunto da literatura. Os dados sobre os conglomerados de relações entre variáveis podem indicar campos de pesquisa e vazios na literatura. Levando-se em consideração a densidade dos referidos grupos, centralidade das variáveis e recorrência de relações entre variáveis, revela-se a "rede de conhecimento" presente na literatura que nas tradicionais revisões integrativas não são possíveis de serem visualizadas.

Portanto, a estratégia apresentada permite identificar "redes de conhecimento" existentes em determinados campos teóricos e verificar lacunas na literatura, a fim de orientar questões de pesquisa. Entretanto, por ser uma estratégia inovadora, a RIRL deve ser colocada em teste em outras temáticas e áreas de conhecimento, visando à identificação de padrões nos achados que possam ser comparados com base na TG e na AR. Neste contexto, dentre as possibilidades que não foram contempladas por esta RIRL, sugere-se a análise de "vazios estruturais", ou seja, a identificação de vazios de relações entre conceitos, variáveis e temáticas.

Destaca-se que nenhum estudo na amostra desta RIRL abordou de forma direta os aspectos relativos à Análise de Redes Sociais (ARS) no que se refere ao ASO, embora todos os estudos estivessem investigando interações sociais. Por se tratar de um fenômeno mais abrangente de rede social, o potencial instrumental da ARS não pode ser negligenciado em pesquisas que tratam de AS, de forma que há uma lacuna quanto à investigação desta variável sob a perspectiva da ARS que deve ser preenchida.

Por fim, verificou-se nesta RIRL que uma sensível proporção das plataformas e das interações descritas se referem a AS em situações de deficiência e/ou condições de saúde. Assim, considerando-se que o ASO diminui o grau de separação entre os envolvidos, sugere-se um maior investimento em pesquisas a fim de conhecer os participantes, tipos de demandas e apoios que circulam em tais redes, o que pode contribuir para um melhor desenvolvimento de políticas públicas a ela relacionadas.

Quanto às limitações gerais desta RIRL, considerando-se a falta de consenso na literatura sobre a definição de AS, é possível que estudos sobre este tema possam estar ausentes devido à indexação sob termos de busca diferentes dos utilizados nesta revisão, embora uma ampla gama de termos correlatos a esta variável tenha sido empregada. Da mesma forma, o uso de literatura advinda unicamente de bases de indexação de periódicos, embora aumente a qualidade dos artigos resgatados, pode ser um fator limitante. Por fim, a predominância do idioma inglês e de publicação em países europeus e norte-americanos também pode ser considerada um fator limitante, o que demanda a verificação de estudos em outros países e idiomas em pesquisas futuras. 


\section{Referências}

Appleton, J., Fowler, C., \& Brown, N. (2014). Friend or foe? An exploratory study of Australian parents' use of asynchronous discussion boards in childhood obesity. Collegian, 21(2), 151-158. doi: 10.1016/j.colegn.2014.02.005.

Asiodu, I.V., Waters, C.M., Dailey, D.E., Lee, K.A., \& Lyndon, A. (2015). Breastfeeding and use of social media among first-time African American mothers. Journal of Obstetric, Gynecologic, \& Neonatal Nursing, 44(2), 268-278. doi: 10.1111/1552-6909.12552.

Binford Hopf, R.B., Grange, D.L., Moessner, M., \& Bauer, S. (2013). Internet-based chat support groups for parents in family-based treatment for adolescent eating disorders: A pilot study. European Eating Disorders Review, 21(3), 215-223. doi: 10.1002/erv.2196.

Brady, E., \& Guerin, S. (2010). "Not the romantic, all happy, coochy coo experience": A qualitative analysis of interactions on an Irish parenting web site. Family Relations, 59(1), 14-27. doi: 10.1111/j.1741-3729.2009.00582.x.

Bragadóttir, H. (2008). Computer-mediated support group intervention for parents. Journal of Nursing Scholarship, 40(1), 32-38. doi: 10.1111/j.1547-5069.2007.00203.x.

Cacioppo, C.N., Conway, L.J., Mehta, D., Krantz, I.D., \& Noon, S.E. (2016). Attitudes about the use of internet support groups and the impact among parents of children with Cornelia de Lange Syndrome. American Journal of Medical Genetics Part C: Seminars in Medical Genetics, 172(2), 229-236. doi: 10.1002/ajmg.c.31504.

Cantwell, J., Muldoon, O.T., \& Gallagher, S. (2014). Social support and mastery influence the association between stress and poor physical health in parents caring for children with developmental disabilities. Research in Developmental Disabilities, 35(9), 2215-2223. doi: 10.1016/j.ridd.2014.05.012.

Clarke, J.N., \& Van Ameron, G. (2015). Parents Whose Children have Oppositional Defiant Disorder Talk to One Another on the Internet. Child and Adolescent Social Work Journal, 32(4), 341-350. doi: 10.1007/s10560-015-0377-5.

Clauset, A., Newman, M.E.J., \& Moore, C. (2004). Finding community structure in very large networks. Physical Review, 70(6), 1-6. doi: 10.1103/PhysRevE.70.066111.

Clifford, T., \& Minnes, P. (2013). Logging on: Evaluating an online support group for parents of children with autism spectrum disorders. Journal of Autism and Developmental Disorders, 43(7), 1662-1675. doi: 10.1007/s10803-012-1714-6.

Coulson, N.S., \& Greenwood, N. (2012). Families affected by childhood cancer: An analysis of the provision of social support within online support groups. Child: Care, Health and Development, 38(6), 870-877. doi:10.1111/j.1365-2214.2011.01316.x.

Craig, E.A., \& Johnson, A.J. (2011). Role strain and online social support for childless stepmothers. Journal of Social and Personal Relationships, 28(6), 868-887. doi: 10.1177/0265407510393055.

Diallo, S.Y., Lynch, C.J., Gore, R., \& Padilla, J.J. (2016). Identifying key papers within a journal via network centrality measures. Scientometrics, 107(3),1005-1020. doi: 10.1007/s11192016-1891-8.

Epifanio, M. S., Genna, V., De Luca, C., Roccella, M., \& La Grutta, S. (2015). Paternal and maternal transition to parenthood: the risk of postpartum depression and parenting stress. Pediatric reports, 7(2), 38-44. doi: 10.4081/pr.2015.5872.

Eriksson, H., \& Salzmann-Erikson, M. (2013). Supporting a caring fatherhood in cyberspace-an analysis of communication about caring within an online forum for fathers. Scandinavian Journal of Caring Sciences, 27(1), 63-69. doi: 10.1111/j.1471-6712.2012.01001.x.

Evans, M., Donelle, L., \& Hume-Loveland, L. (2012). Social support and online postpartum depression discussion groups: A content analysis. Patient Education and Counseling, 87(3), 405-410. doi: 10.1016/j.pec.2011.09.011. 
Fletcher, R., \& StGeorge, J. (2011). Heading into fatherhood-nervously: Support for fathering from online dads. Qualitative Health Research, 21(8), 1101-1114. doi: $10.1177 / 1049732311404903$.

Gabbert, T.I., Metze, B., Bührer, C., \& Garten, L. (2013). Use of social networking sites by parents of very low birth weight infants: experiences and the potential of a dedicated site. European Journal of Pediatrics, 172(12), 1671-1677. doi: 10.1007/s00431-013-2067-7.

Gallagher, S., \& Whiteley, J. (2012). Social support is associated with blood pressure responses in parents caring for children with developmental disabilities. Research in Developmental Disabilities, 33(6), 2099-2105. doi: 10.1016/j.ridd.2012.06.007.

Glenn, A.D. (2015). Using online health communication to manage chronic sorrow: Mothers of children with rare diseases speak. Journal of Pediatric Nursing, 30(1), 17-24. doi: 10.1016/j.pedn.2014.09.013.

Hall, W., \& Irvine, V. (2009). E-communication among mothers of infants and toddlers in a community-based cohort: A content analysis. Journal of Advanced Nursing, 65(1), 175183. doi: 10.1111/j.1365-2648.2008.04856.x.

Hanneman, R., \& Riddle, M. (2005). Introduction to social network methods. Riverside, CA, EUA: University of California.

Hudson, D.B., Campbell-Grossman, C., \& Hertzog, M. (2012). Effects of an internet intervention on mothers' psychological, parenting, and health care utilization outcomes. Issues in Comprehensive Pediatric Nursing, 35(3-4), 176-193. doi: 10.3109/01460862.2012.734211.

Jacobs, R., Boyd, L., Brennan, K., Sinha, C.K., \& Giuliani, S. (2016). The importance of social media for patients and families affected by congenital anomalies: A Facebook crosssectional analysis and user survey. Journal of Pediatric Surgery, 51(11), 1766-1771. doi: 10.1016/j.jpedsurg.2016.07.008.

Johnson, S.A. (2015). 'Intimate mothering publics': comparing face-to-face support groups and Internet use for women seeking information and advice in the transition to first-time motherhood. Culture, Health \& Sexuality, 17(2), 237-251. doi: 10.1080/13691058.2014.968807.

Kirk, S., \& Milnes, L. (2016). An exploration of how young people and parents use online support in the context of living with cystic fibrosis. Health Expectations, 19(2), 309-321. doi: 10.1111/hex.12352.

Laniado, D., Volkovich, Y., Kappler, K., \& Kaltenbrunner, A. (2016). Gender homophily in online dyadic and triadic relationships. EPJ Data Science, 5(19), 1-23. doi: 10.1140/epjds/s13688-016-0080-6.

Lee, R.L., \& Kvasny, L. (2014). Understanding the role of social media in online health: A global perspective on online social support. First Monday, 19(1). doi: 10.5210/fm.v19i1.4048.

Lewis, T.G. (2009). Network science: Theory and practice. Hoboken, NJ, EUA: John Wiley \& Sons, Inc.

Lin, T.C., Hsu, J.S.C., Cheng, H.L., \& Chiu, C.M. (2015). Exploring the relationships between receiving and offering online social support: A dual social support model. Information \& Management, 52(3), 371-383. doi: 10.1016/j.im.2015.01.003.

Lovell, B., Moss, M., \& Wetherell, M.A. (2012). With a little help from my friends: Psychological, endocrine and health corollaries of social support in parental caregivers of children with autism or ADHD. Research in Developmental Disabilities, 33(2), 682-687. doi: 10.1016/j.ridd.2011.11.014.

Martin, S., Roderick, M.C., Lockridge, R., Toledo-Tamula, M.A., Baldwin, A., Knight, P., \& Wolters, P. (2016). Feasibility and preliminary efficacy of an internet support group for parents of a child with neurofibromatosis type 1: a pilot study. Journal of genetic counseling, 26(3), 576-585. doi: 10.1007/s10897-016-0031-1. 
Martin, S., Wolters, P.L., Baldwin, A., Roderick, M.C., Toledo-Tamula, M.A., Gillespie, A., \& Widemann, B. (2014). Attitudes about internet support groups among adolescents and young adults with neurofibromatosis type 1 and their parents. Journal of Genetic Counseling, 3(26), 576-585. doi: 10.1007/s10897-016-0031-1.

Mendes, K.D.S., Silveira R.C.C.P., \& Galvão, C.M. (2008). Revisão integrativa: método de pesquisa para a incorporação de evidências na saúde e na enfermagem. Texto Contexto Enfermagem, 17(4), 758-64. Recuperado de http://www.redalyc.org/html/714/71411240017/.

Morris, H., \& Bertram, D. (2013). Therapist utilization of online social support for parents of premature infants. Contemporary Family Therapy, 35(3), 583-598. doi: 10.1007/s10591013-9239-5.

Mustafa, H.R., Short, M., \& Fan, S. (2015). Social support exchanges in Facebook social support group. Procedia-Social and Behavioral Sciences, 185, 346-351. doi: 10.1016/j.sbspro.2015.03.449.

Niela-Vilén, H., Axelin, A., Salanterä, S., \& Melender, H. L. (2014). Internet-based peer support for parents: A systematic integrative review. International Journal of Nursing Studies, 51(11), 1524-1537. doi: 10.1016/j.ijnurstu.2014.06.009.

Nolan, S., Hendricks, J., \& Towell, A. (2015). Social networking sites (SNS): exploring their uses and associated value for adolescent mothers in Western Australia in terms of social support provision and building social capital. Midwifery, 31(9), 912-919. doi: 10.1016/j.midw.2015.05.002.

Oprescu, F., Campo, S., Lowe, J., Andsager, J., \& Morcuende, J. (2015). Online information exchanges for parents of children with a rare health condition: Key findings from an online support community. Journal of Medical Internet Research, 15(1), e16. doi:10.2196/jmir.2423.

Park, E., Kim, H., \& Steinhoff, A. (2016). Health-related internet use by informal caregivers of children and adolescents: an integrative literature review. Journal of Medical Internet Research, 18(3), e57. doi: 10.2196/jmir.4124.

Parry, D.C., Glover, T.D., \& Mulcahy, C.M. (2013). From" stroller-stalker" to" momancer" courting friends through a social networking site for mothers. Journal of Leisure Research, 45(1), 23-46. doi: 10.18666/jlr-2013-v45-i1-2941.

Pedersen, S., \& Lupton, D. (in press). 'What are you feeling right now? 'communities of maternal feeling on Mumsnet. Emotion, Space and Society. doi: 10.1016/j.emospa.2016.05.001.

Perrone, M.E., Carmody, D., Philipson, L.H., \& Greeley, S.A.W. (2015). An online monogenic diabetes discussion group: supporting families and fueling new research. Translational Research, 166(5), 425-431. doi: 10.1016/j.trsl.2015.06.013.

Porter, N., \& Ispa, J.M. (2013). Mothers' online message board questions about parenting infants and toddlers. Journal of Advanced Nursing, 69(3), 559-568. doi: 10.1111/j.13652648.2012.06030.x.

Ramos, M.F.H., Fernandez, A.P.O., Pontes, F.A.R., \& Silva, S.S.C. (2016). Caracterização das pesquisas sobre eficácia coletiva docente na perspectiva da teoria social cognitiva. Psicologia: Teoria e Pesquisa, 32(1), 91-99. doi: 10.1590/0102-37722016012227091099.

Reinke, J.S., \& Solheim, C.A. (2015). Online social support experiences of mothers of children with autism spectrum disorder. Journal of Child and Family Studies, 24, 2364-2373. doi: 10.1007/s10826-014-0039-9.

Roffeei, S.H.M., Abdullah, N., \& Basar, S.K.R. (2015). Seeking social support on Facebook for children with Autism Spectrum Disorders (ASDs). International Journal of Medical Informatics, 84(5), 375-385. doi: 10.1016/j.ijmedinf.2015.01.015. 
Salzmann-Erikson, M., \& Eriksson, H. (2013). Fathers sharing about early parental support in health-care-online discussions on an Internet forum. Health \& Social Care in the Community, 21(4), 381-390. doi: 10.1111/hsc.12028.

Stewart, M., Letourneau, N., Masuda, N.R., Anderson, S., \& McGhan, S. (2011). Online solutions to support needs and preferences of parents of children with asthma and allergies. Journal of Family Nursing, 17(3), 357-379. doi: 10.1177/1074840711415416.

Sullivan, C.F. (2008). Cybersupport: Empowering asthma caregivers. Pediatric Nursing, 34(3), 217-224. Recuperado de https://www.ncbi.nlm.nih.gov/pubmed/18649811.

Thuy, N.T.M., \& Berry, H.L. (2013). Social capital and mental health among mothers in Vietnam who have children with disabilities. Global Health Action, 6, 254-260. doi: 10.3402/gha.v6i0.18886.

Valtchanov, B.L., Parry, D.C., Glover, T.D., \& Mulcahy, C.M. (2014). Neighborhood at your fingertips: Transforming community online through a Canadian social networking site for mothers. Gender, Technology and Development, 18(2), 187-217. doi: 10.1177/0971852414529481.

Wei, Y.S., Chu, H., Chen, C.H., Hsueh, Y.J., Chang, Y.S., Chang, L.I., \& Chou, K.R. (2012). Support groups for caregivers of intellectually disabled family members: Effects on physical-psychological health and social support. Journal of Clinical Nursing, 21(11-12), 1666-1677. doi: 10.1111/j.1365-2702.2011.04006.x.

Whittemore, R., \& Knafl, K. (2005). The integrative review: update methodology. Journal of Advanced Nursing, 52(5), 546-53. doi: 10.1111/j.1365-2648.2005.03621.x.

Yep, J., \& Shulman, J. (2014). Analyzing the library's Twitter network: Using NodeXL to visualize impact. College \& Research Libraries News, 75(4), 177-186. Recuperado de http://crln.acrl.org/content/75/4/177.full.

Financiamento: A realização deste estudo foi financiada, em parte, pela Coordenação de Aperfeiçoamento de Pessoal de Nível Superior - Brasil (CAPES) - Edital 59/2014 (Tecnologia Assistiva) e a sua tradução para a lingua inglesa foi financiada pela Pró-Reitoria de Pesquisa e Pós-Graduação (PROPESP) da Universidade Federal do Pará (UFPA)- Edital 01/2020.

Participação dos autores: a) Planejamento e concepção do trabalho; b) Coleta de dados; c) Análise e interpretação de dados; d) Redação do manuscrito; e) Revisão crítica do manuscrito.

T.S.D. contribuiu em a, b, c, d, e; F.A.R.P. em a, c, e; H.C.A. em b, c; T.S.R. em b, c; M.F.H.R. em e; S.S.C.S. em e.

Editora científica responsável: Dra. Cecilia Cracco 